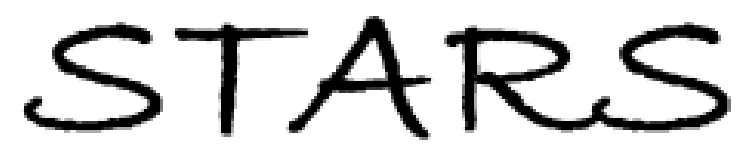

University of Central Florida

STARS

$1-1-1998$

\title{
Phase diagram of three-dimensional dynamical triangulations with a boundary
}

Simeon Warner

Simon Carrerall

Ray Renken

University of Central Florida

Find similar works at: https://stars.library.ucf.edu/facultybib1990

University of Central Florida Libraries http://library.ucf.edu

This Article is brought to you for free and open access by the Faculty Bibliography at STARS. It has been accepted for inclusion in Faculty Bibliography 1990s by an authorized administrator of STARS. For more information, please contact STARS@ucf.edu.

\section{Recommended Citation}

Warner, Simeon; Carrerall, Simon; and Renken, Ray, "Phase diagram of three-dimensional dynamical triangulations with a boundary" (1998). Faculty Bibliography 1990s. 2490.

https://stars.library.ucf.edu/facultybib1990/2490

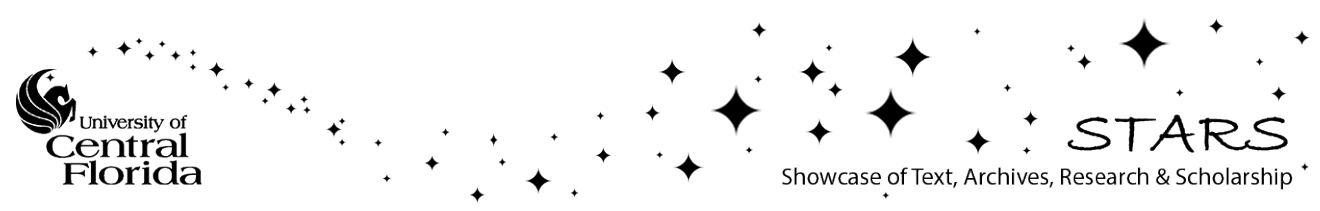




\title{
Phase diagram of three-dimensional dynamical triangulations with a boundary
}

\author{
Simeon Warner ${ }^{\mathrm{a}, 1}$, Simon Catterall ${ }^{\mathrm{a}}$, Ray Renken ${ }^{\mathrm{b}}$ \\ ${ }^{a}$ Department of Physics, Syracuse University, Syracuse, NY 13210, USA \\ ${ }^{\mathrm{b}}$ Department of Physics, University of Central Florida, Orlando, FL 32816, USA
}

Received 30 July 1998; revised 8 September 1998

Editor: P.V. Landshoff

\begin{abstract}
We use Monte Carlo simulation to study the phase diagram of three-dimensional dynamical triangulations with a boundary. Three phases are indentified and characterized. One of these phases is a new, boundary dominated phase; a simple argument is presented to explain its existence. First-order transitions are shown to occur along the critical lines separating phases. (C) 1998 Elsevier Science B.V. All rights reserved.
\end{abstract}

\section{Dynamical triangulations with a boundary term}

Dynamical triangulation models arise from simplicial discretizations of continuous Riemannnian manifolds. A manifold is approximated by glueing together a set of equilateral simplices with fixed edgelengths. This glueing ensures that each face is shared by exactly two distinct simplices - the resultant simplicial lattice is called a triangulation. In the context of Euclidean quantum gravity it is natural to consider a weighted sum of all possible triangulations as a candidate for a regularized path integral over metrics. Physically distinct metrics correspond

\footnotetext{
${ }^{1}$ E-mail: simeon@physics.syr.edu
}

to inequivalent simplicial triangulations. This prescription has been shown to be very successful in two-dimensions (see, for example [1],).

Most analytic studies and almost all numerical work done so far has been restricted to compact manifolds like the sphere. In this paper we develop techniques that allow us to extend numerical studies to simplicial manifolds with boundaries. Our work is, in part, motivated by the possibility of computing an object which can be thought of as a simplicial analog of the 'wavefunction of the Universe' [2]:

$$
\psi[h]=\int D g e^{-S(g)}
$$

The functional integral over 3-metrics $g$ is restricted to those with 2-metric $h$ on the boundary. We study the 3-disk created by inserting an $S^{2}$ boundary into a 
triangulation of the sphere $S^{3}$. Fixing $h$ corresponds to choosing a particular boundary 2-triangulation $T_{2}$, and the simplicial wavefunction is:

$$
\psi\left(T_{2}\right)=\sum_{T_{3}} e^{-S_{L}\left(T_{3}\right)}
$$

The probability amplitude for finding a particular 2-triangulation $T_{2}$ is obtained by counting (with some weight) all 3-triangulations $T_{3}$ which contain $T_{2}$ as their boundary. In this work we do not attempt to calculate $\psi\left(T_{2}\right)$ for particular $T_{2}$. However, we do calculate the probability of finding 3-triangulations with particular boundary area

$p(A)=\sum_{T_{2}} \psi\left(T_{2}\right) \delta_{N_{2}\left(T_{2}\right), A}$

where $A$ is the area of the boundary, equal to the number of triangles $N_{2}\left(T_{2}\right)$. We use the mean of $p(A)$ as an order parameter to characterize the phases of the model. Several caveats are in order. First, our simulations are restricted to fixed $T_{3}$ volume. Second, the lattice action we will introduce is bounded from below unlike the continuum Einstein-Hilbert action. Third, we have no proof that our sum over simplicial lattices correctly reproduces the integration over physically inequivalent metrics.

A natural lattice action $S_{L}$ can be derived from the continuum action by straightforward techniques [3]. It contains both the usual Regge curvature piece familiar from compact triangulations together with a boundary term. The boundary term arises from discretization of the extrinsic curvature of the boundary embedded in the bulk. In three-dimensions the curvature is localized on links. If $L_{M}$ denotes the set of links in the bulk of the 3-triangulation (excluding the boundary) and $L_{\partial M}$ those in the boundary the action can be written

$S_{L}=\kappa_{1}\left(\sum_{h \in L_{M}}\left(2 \pi-\alpha n_{h}\right)+\sum_{h \in L_{\partial M}}\left(\pi-\alpha n_{h}\right)\right)$

The quantity $\alpha=\arccos (1 / 3)$ and $n_{h}$ is the number of simplices sharing the link (hinge) $h$. Typically $S_{L}$ will also contain a bulk cosmological constant that can be used to tune the simulation volume. The resultant action can be rewritten in the form

$S_{b}=-\kappa_{0} N_{0}+\kappa_{3} N_{3}+\kappa_{b} N_{2}^{b}$

where $N_{2}^{b}$ is the area of the boundary. Here, $\kappa_{3}$ is used to tune the volume of the system. We are thus left with a two-dimensional phase space parameterized by $\kappa_{0}$ and $\kappa_{b}$ conjugate to the number of vertices and the number of boundary triangles. It is trivial to generalize this to, for example, four-dimensions. The partition function for the system is then

$Z=\sum_{T} e^{-S_{b}}$

where the sum is over triangulations, $T$.

Various other extended phase diagrams have been studied for three-dimensional dynamical triangulations including adding spin matter [4,5], adding gauge matter [6,7], and adding a measure term [8]. Much of this work was motivated by the desire to find a continuous phase transition. No such transitions have been found.

\section{Simulation}

Our simulation algorithm is an extension of the algorithm for compact manifolds in arbitrary dimension described by Catterall [9]. Consider the environment of any vertex in a $D$-triangulation - it is composed of simplices making up a trivial $D$-ball. The boundary of this $D$-ball is just the sphere $S^{(D-1)}$. A boundary with the topology of $S^{(D-1)}$ can thus be created in the original triangulation by removing these simplices. If the original triangulation corresponded to the sphere $S^{D}$ the topology of the new triangulation is that of a $D$-disk.

In practice we simulate a compact manifold with one 'special' vertex. This vertex and all the simplices sharing it are ignored during any measurement. In this way every triangulation of our marked sphere $S^{D}$ is in one-to-one correspondence with a triangulation of the $D$-disk. Notice that the usual compact manifold moves applied to all simplices (including those sharing the special vertex) will in 
general change the boundary of the $D$-disk. Indeed these moves are ergodic with respect to the boundary. Furthermore, the proof that these moves satisfy a detailed balance relation goes through just as for the compact case. The one extra restriction is simple - one must never delete the special vertex. With this trick we can trivially extend our compact codes to the situation in which a $S^{(D-1)}$ boundary has been added. We are merely simulating a compact lattice with an action which singles out a special vertex and its neighbour simplices. This contrasts with the set of additional boundary moves used by Adi et al. [10] for simulations in two-dimensions.

Measurements do not include the special vertex or any simplices connected to it. For example, the number of $D$-simplices in the system with boundary is the number of $D$-simplices in the whole simulation minus the number of $D$-simplices sharing the special vertex. The size of the boundary is simply the number of $D$-simplices sharing the special vertex.

We have used the Metropolis Monte Carlo [11] scheme with usual update rule:

$p($ accept move $)=\min \left\{e^{-\Delta S_{b}}, 1\right\}$

and in this way we explore the space of unlabeled triangulations with the action $S_{b}$ (Eq. (5) for threedimensions).

\subsection{Checks in two-dimensions}

In two dimensions we tested our simulation code at small volumes by comparing with hand calculated amplitudes for small disks. We label disk configurations by the number of triangles and the boundary

Table 1

Estimates of $\left\langle N_{2}^{b}\right\rangle$, the expectation value of the boundary size (length), for two-dimensional manifolds of various sizes from Adi et al. [10], and from this work

\begin{tabular}{lcc}
\hline$N_{3}$ & $\left\langle N_{2}^{b}\right\rangle$ (Adi et al.) & $\left\langle N_{2}^{b}\right\rangle$ (this work) \\
\hline 50 & $39.83(5)$ & $39.88(4)$ \\
100 & $78.05(5)$ & $78.09(4)$ \\
200 & $154.54(6)$ & $154.57(6)$ \\
400 & $307.62(8)$ & $307.53(8)$ \\
800 & $613.4(1)$ & $613.4(1)$ \\
1600 & $1225.1(1)$ & $1225.1(2)$ \\
3200 & $2448.7(2)$ & $2448.8(3)$ \\
6400 & $4895.6(3)$ & $4895.4(13)$ \\
\hline
\end{tabular}

length: $\left(N_{2}, N_{1}^{b}\right)$. We calculated the ratios of amplitudes for disks $(1,1):(2,4):(3,3):(3,5)$ to be $1: 1.5: 1: 3$. Our simulation gave 1.01:1.52:1:3.04 from a sample of 1 million disks with volume 1-3. This test was extended up to volume 5 disks, also showing good agreement.

We also tested our simulation code by comparing results for two-dimensions of Adi et al. [10]. All our results agree within the statistical errors. Table 1 shows a comparison of the results for a selection of lattice sizes.

\section{Phase diagram}

We performed a set of simulations in three-dimensions with action of Eq. (5). In all runs $\kappa_{3}$ was used to tune the nominal system volume, $N_{3}$, to 2000 for each given $\kappa_{0}$ and $\kappa_{b}$.

In three-dimensions there are just 4 types of move: vertex insertion, vertex deletion and exchange of a link with a face (two moves: link to face or face to link). Where these moves take place on sections of the triangulation involving the special vertex we take care to count changes in the numbers of simplices inside and outside of the boundary but otherwise the moves are the same as for the bulk. Series of runs varying either $\kappa_{0}$ or $\kappa_{b}$ were made and the vertex susceptibility used to search for phase transitions. We define the vertex susceptibility, $\chi$, to be normalized with respect to the number of 3-simplices:

$\chi=\frac{1}{N_{3}}\left(\left\langle N_{0}^{2}\right\rangle-\left\langle N_{0}\right\rangle^{2}\right)$

The points shown in Fig. 1 are taken from the positions of peaks in the vertex susceptibility.

In Fig. 1 there are three phases which we characterize as: phase 1 - crumpled, minimal boundary; phase 2 - branched-polymer, minimal boundary; and phase 3 - boundary dominated. In phases 1 and 2 the boundary is simply 4 triangles (2-simplices) connected to form a tetrahedral hole. The system is essentially like a compact manifold with one marked 3 -simplex - the tetrahedral hole. In phase 3 the boundary is large - typically a substantial fraction of the bulk volume. 


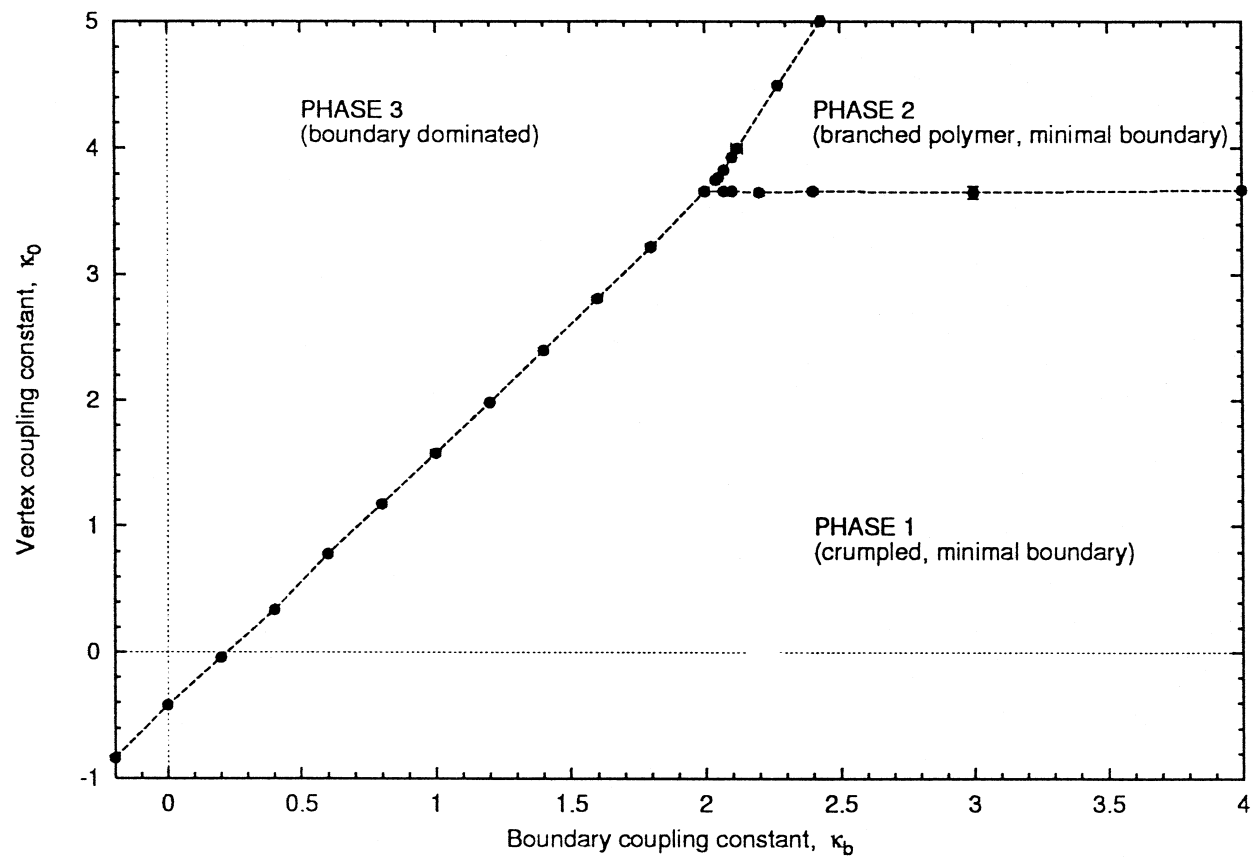

Fig. 1. Phase diagram for 3-dimensional dynamical triangulation with a boundary. All points have error bars in either $\kappa_{b}$ or $\kappa_{0}$, most cannot be seen because they are smaller than the symbols. Nominal simulation volume, $N_{3}=2000$.

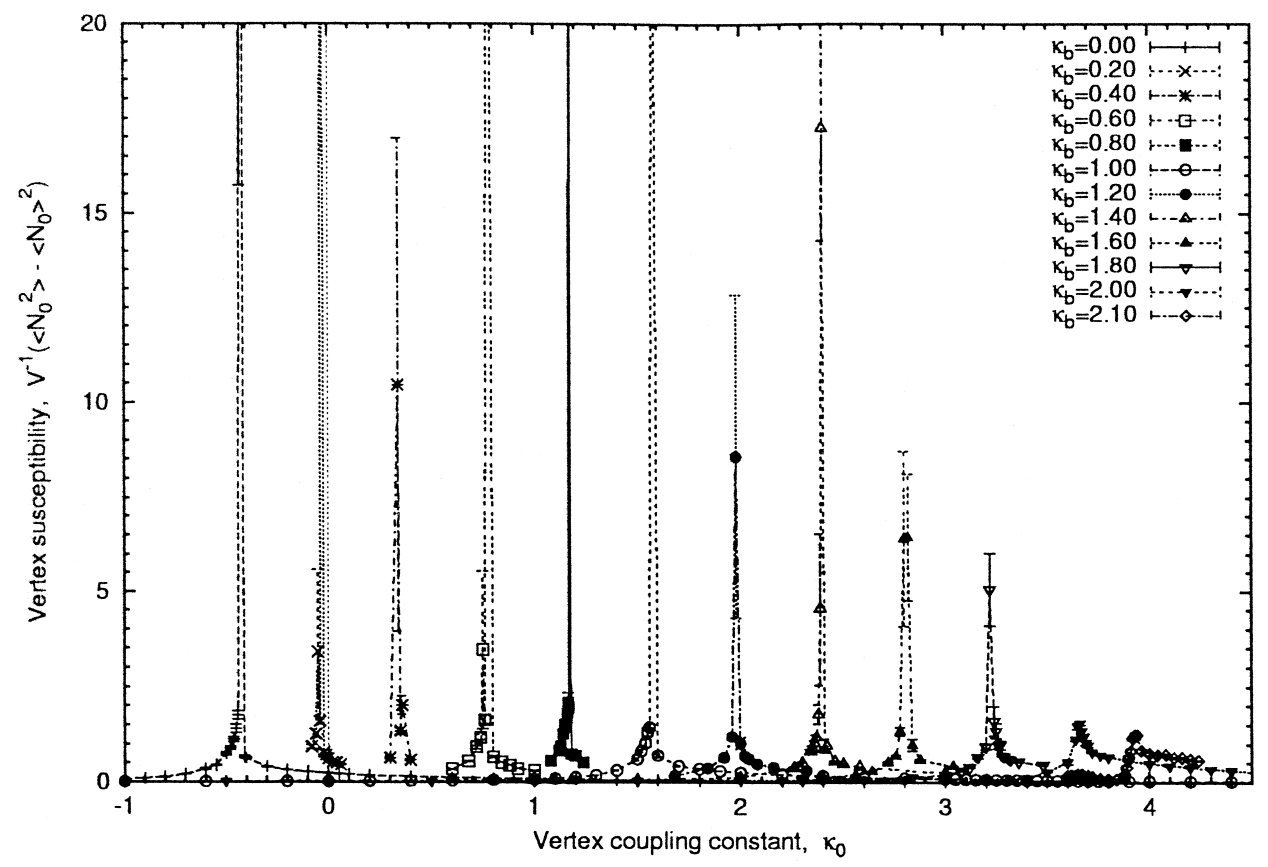

Fig. 2. Sample of vertex susceptibility data for different values of the boundary coupling constant, $\kappa_{b}$. 


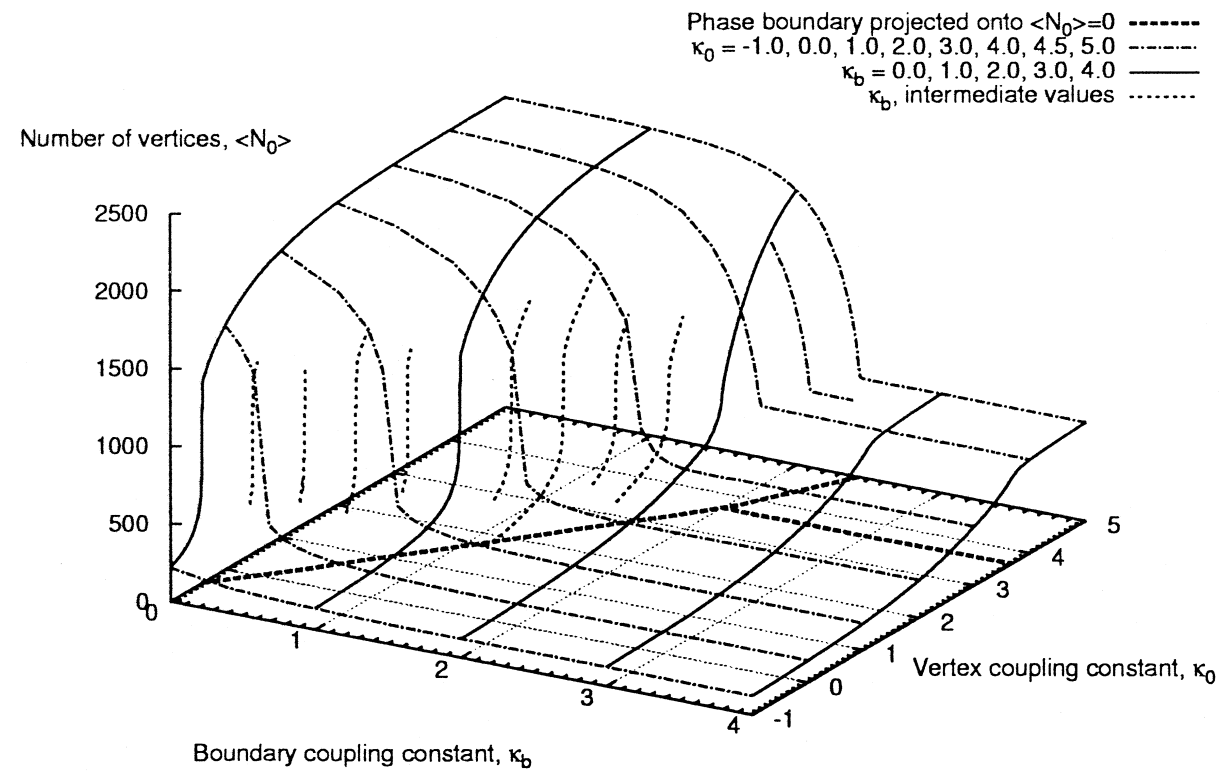

Fig. 3. Number of vertices, $\left\langle N_{0}\right\rangle$, as a function of $\kappa_{0}$ and $\kappa_{b}$. Nominal simulation volume, $N_{3}=2000$. Note that we see three distinct areas with different values of $\left\langle N_{0}\right\rangle$ : the boundary dominated phase (small $\kappa_{b}$, large $\kappa_{0}$ ) with large $\left\langle N_{0}\right\rangle$, the crumpled phase (small $\kappa_{0}$ ) with small $\left\langle N_{0}\right\rangle$, and the branched-polymer phase (large $\kappa_{b}$ and $\kappa_{0}$ ) with intermediate $\left\langle N_{0}\right\rangle$.
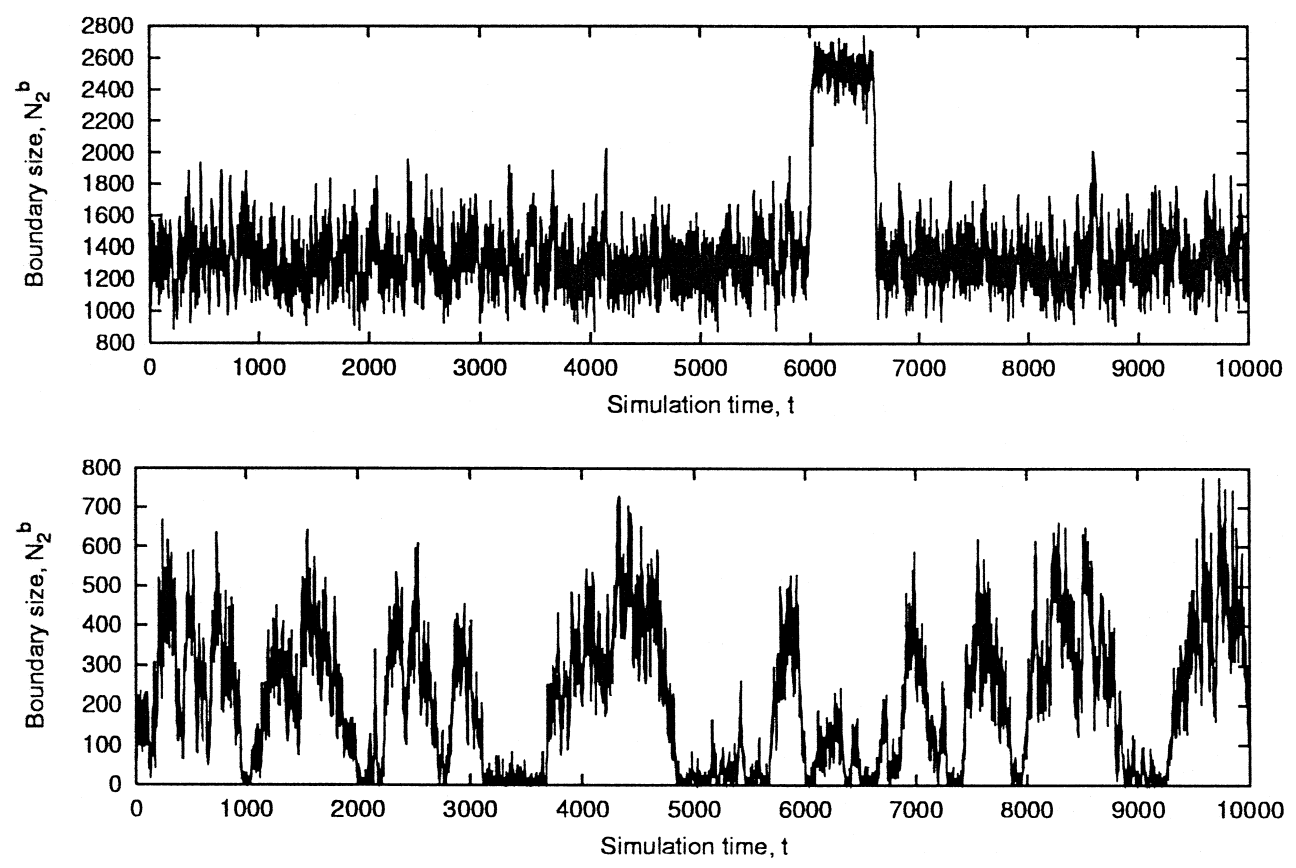

Fig. 4. Time series showing the boundary size $\left(N_{2}^{b}\right)$ during simulation. The upper plot is at the transition between the crumpled and boundary dominated phases $\left(k_{0}=-0.423, k_{b}=0\right)$. The lower plot is at the transition between the branched-polymer and boundary dominated phases $\left(k_{0}=5, k_{b}=2.43\right)$. Nominal simulation volume, $N_{3}=2000$, and time is in units of $100 N_{3}$ attempted updates. 


\subsection{Simple argument for boundary dominated phase}

Here we argue that the boundary dominated phase can be explained by considering an effective action written in terms of the boundary size. We show that in certain circumstances a large boundary will decrease this action. Otherwise one of the minimal boundary phases will be favored.

Consider the action:

$S_{b}=-k_{0} N_{0}+k_{b} N_{2}^{b}$

We ignore the volume term as this is kept fixed during the simulation. If we note that the boundary is itself a 2-sphere then we know that:

$N_{2}^{b}=2\left(N_{0}^{b}-2\right)$

and

$N_{0}=N_{0}^{b}+N_{0}^{i}$

where $N_{0}$ is the number of vertices, $N_{0}^{b}$ is the number of vertices on the boundary, $N_{0}^{i}$ is the number of internal vertices, and $N_{2}^{b}$ is the number of 2 -simplices (triangles) on the boundary. We may thus rewrite the action:

$S_{b}=-\kappa_{0} N_{0}^{i}+\left(2 \kappa_{b}-\kappa_{0}\right) N_{0}^{b}$

If we now consider $N_{0}^{i}$ fixed and note that the number of manifolds with boundary size $N_{2}^{b}$ is governed by an exponential factor $e^{\kappa_{b}^{c} N_{2}^{b}}=e^{2 \kappa_{b}^{c} N_{0}^{b}}$, where $\kappa_{b}^{c}$ is a new constant, we may then write an effective action for the number of boundary vertices:

$S_{\text {eff }} \approx\left(-\kappa_{0}+2\left(\kappa_{b}-\kappa_{b}^{c}\right)\right) N_{0}^{b}$

The presence of small or large boundaries is then determined by the sign of this action. We thus expect the phase transition at $\kappa_{0}=2\left(\kappa_{b}-\kappa_{b}^{c}\right)$ which is in good agreement with what we see (Fig. 2 and Fig. $3)$.

\subsection{Order of transitions}

Simulations of compact manifolds in three and four-dimensions are known to have a first-order phase transition between crumpled and branched polymer phases (3d [12], 4d [13,14]). Our Monte Carlo time series show strong bistability on all three phase boundaries (see Fig. 4). We take this to indicate that all three phase transitions are first-order.

\section{Concluding remarks}

We have demonstrated an arbitrary dimension algorithm for simulating dynamical triangulations with a boundary. This has been tested against known results in two-dimensions and used to map the phase diagram in three-dimensions.

We have identified three phases in three-dimensional dynamical triangulations with a boundary and mapped the boundaries within the range of couplings $-1<\kappa_{0}<5$ and $-0.5<\kappa_{b}<4$. The observed phases include the crumpled and branched-polymer phases seen in triangulations of compact manifolds, and also a new, boundary dominated phase. The existence of this phase, and the shape of the phase boundary on the $\kappa_{0}-\kappa_{b}$ phase diagram, is predicted by a simple argument. Obvious bistability in the time series at the phase transitions indicates that all transitions within the range of couplings studied are firstorder.

\section{Acknowledgements}

Simon Catterall was supported in part by DOE grant DE-FG02-85ER40237. Ray Renken was supported in part by NSF grant PHY-9503371.

\section{References}

[1] J. Ambjorn, XXX archive, hep-th/9411179, 1994.

[2] J.B. Hartle, S.W. Hawking, Physical Review D 28 (1983) 2960.

[3] J.B. Hartle, R. Sorkin, General Relativity and Gravity 13 (1981) 541

[4] R.L. Renken, S.M. Catterall, J.B. Kogut, Nuclear Physics B 422 (1994) 677.

[5] J. Ambjorn, C. Kristjansen, Z. Burda, J. Jurkiewicz, Nuclear Physics B (Proc. Suppl.) (1993) 771.

[6] R.L. Renken, S.M. Catterall, J.B. Kogut, Nuclear Physics B 389 (1993) 601.

[7] J. Ambjorn, J. Jurkiewicz, S. Bilke, Z. Burda, B. Petersson, Modern Physics Letters A 9 (1994) 2527.

[8] R.L. Renken, S.M. Catterall, J.B. Kogut, XXX archive, hep-lat/9712011, 1997. 
[9] S. Catterall, Computer Physics Communications 87 (1995) 409.

[10] E. Adi, M. Hasenbusch, M. Marcu, E. Pazy, K. Pinn, S. Solomon. XXX archive, hep-lat/9310016, 1993.

[11] N. Metropolis, A.W. Rosenbluth, M.N. Rosenbluth, A.H. Teller, E. Teller, The Journal of Chemical Physics 21 (1953).
[12] J. Ambjorn, D.V. Boulatov, A. Krzywicki, S. Varsted, Physiscs Letters B 276 (1992) 432.

[13] P. Bialas, Z. Burda, A. Krzywicki, B. Petersson, Nuclear Physics B 472 (1996) 293.

[14] B. de Bakker, Physiscs Letters B 389 (1996) 238. 